FORMATION Formation emploi

Revue française de sciences sociales

109 | janvier-mars 2010

L'orientation scolaire et professionnelle dans un monde incertain

\title{
Orientation et politiques publiques
}

Évolutions nationales, enjeux internationaux

Career guidance and public policy: National trends, international issues

Ausbildungs- und Berufsorientierung und öffentliche Politiken : Nationale

Entwicklungen, internationale Herausforderungen

Orientación y políticas públicas : Evoluciones nacionales, desafíos

internacionales

\section{Isabelle Borras et Claudine Romani}

\section{(2) OpenEdition}

Journals

Édition électronique

URL : https://journals.openedition.org/formationemploi/2847

DOI : $10.4000 /$ formationemploi.2847

ISSN : 2107-0946

Éditeur

La Documentation française

Édition imprimée

Date de publication : 15 mars 2010

Pagination : $9-22$

ISSN : 0759-6340

Référence électronique

Isabelle Borras et Claudine Romani, «Orientation et politiques publiques », Formation emploi [En ligne], 109 | janvier-mars 2010, mis en ligne le 01 mars 2012, consulté le 28 juin 2022. URL : http://

journals.openedition.org/formationemploi/2847 ; DOI : https://doi.org/10.4000/formationemploi.2847

(C) Tous droits réservés 


\title{
DOSSIER
}

\section{Orientation et politiques publiques}

\section{Évolutions nationales, enjeux internationaux}

Isabelle Borras et Claudine Romani*

\begin{abstract}
L'analyse historique souligne la cohérence entre développement des services d'orientation, politiques éducatives et transformations du marché du travail en France. Ce développement est aujourd'hui marqué par l'influence du contexte international et des effets de l'action communautaire.
\end{abstract}

Parce qu'elle contribue à toutes les politiques publiques d'emploi et de formation, l'orientation n'a jamais vraiment été constituée en secteur autonome de l'action publique. Ceci est actuellement remis en cause, sous l'effet conjugué des nouvelles initiatives des instances internationales, dont l'Union européenne et l'OCDE (Organisation de coopération et de développement économiques), et en raison d'un nouveau contexte du marché du travail. Des préconisations au plan international prônent l'instauration d'« une orientation tout au long de la vie » et appellent à un changement des structures et des modes de fonctionnement existants ${ }^{1}$.

\footnotetext{
${ }^{1}$ La première conférence de la présidence française de l’Union européenne, organisée par le MENESR (ministère de l'Éducation nationale, de l'Enseignement supérieur et de la Recherche), les 17-18 septembre 2008 à Lyon, avait pour objet emblématique : "Gérer les transitions : l'orientation tout au long de la vie dans l'espace européen ». Elle visait à mesurer les progrès dans la mise en œuvre des politiques et principes arrêtés par l’Union européenne et à faire apparaître les convergences.
}

* Isabelle Borras, économiste, est ingénieur de recherches au Lepii-Cnrs (Laboratoire d'économie de la production et de l'intégration internationale), centre associé Céreq, à l'université Pierre Mendès France (Grenoble). Ses travaux portent sur la gouvernance des politiques d'orientation, de formation et d'emploi et interrogent les liens entre action publique et marché du travail.

Claudine Romani, économiste, est chargée de mission au Céreq et y anime la plate-forme d'études sur l'orientation tout au long de la vie. Elle est spécialiste de la comparaison internationale des marchés du travail et de l'évaluation des politiques -publiques d'emploi et de formation.

Sur le thème de l'orientation, elles ont publié récemment (2009), "La qualité de l'orientation en débat ", BrefCéreq, $n^{\circ} 264,4$ p. ; (2008), "Le pilotage de l'orientation tout au long de la vie: le sens des réformes", Collection Notes Emploi Formation, Céreq, $n^{\circ} 29$; (2008), " Les choix d'orientation à l'épreuve de l'emploi », Céreq, Collection Notes Emploi Formation, $n^{\circ} 30$. 
Malgré une abondance de travaux consacrés aux pratiques et aux dispositifs d'orientation, les réflexions de nature institutionnelle, aptes à saisir les enjeux des réformes en cours, sont encore rares ; il en va de même des études abordant de manière globale l'orientation des jeunes et des adultes. Cette rareté relative contraste nettement avec l'ampleur des mouvements de réforme en Europe, interrogeant les organisations et les régulations héritées de l'histoire. À partir d'une réflexion sur le cas de la France, mis en perspective avec d'autres pays étrangers, cet article propose d'analyser le sens des réformes en trois temps.

La première partie porte un regard historique sur le développement des services d'orientation en France, saisis dans leur cohérence avec les politiques d'éducation et d'emploi. Constitués progressivement dans le temps, trois segments cloisonnés coexistent à ce jour et se distinguent par le statut des publics auxquels ils s'adressent : scolaires, demandeurs d'emplois et salariés. Dans le cadre de la mise en œuvre d'une politique d'orientation tout au long de la vie, suggérée par les instances communautaires, cette segmentation pose aujourd'hui deux questions fondamentales, objets des réformes en cours. La première est celle de la qualité et la seconde celle du décloisonnement des services d'orientation.

À partir d'une revue de la littérature, la deuxième et la troisième partie traitent de la façon dont sont abordés ces deux enjeux d'action publique, en France et à l'étranger. En France, la relative indétermination de la répartition des responsabilités entre l’État et les Régions, sur l'orientation, soulève des problèmes particuliers quant à la conduite des réformes. Les exemples étrangers illustrent les modalités de réponse apportées au double problème de l'amélioration de la qualité et de la coordination des services.

\section{LA NAISSANCE DES TROIS SEGMENTS DE L'ORIENTATION EN FRANCE}

Un survol historique des politiques et des services d'orientation, en France, est riche d'enseignements, notamment sur leur rôle dans une régulation du marché du travail. Quatre grandes phases d'évolution peuvent être distinguées : la naissance de services publics spécialisés d'orientation en formation, la généralisation des services publics d'orientation et leur dilution au sein des intervenants de l'emploi, l'amorce d'une privatisation en lien avec l'orientation des salariés, et l'européanisation du cadre national. À chacune de ces phases correspondent une problématique liée à l'état du marché du travail, un type de réponses spécifique apporté par les politiques de formation et d'emploi, ainsi qu'une structuration des services d'orientation venant en outiller la mise en œuvre².

\section{La spécialisation (1960/1970)}

Le trait dominant de cette première phase est la constitution de services publics spécialisés accompagnant des politiques d'élévation des niveaux de qualification de la population active. Trois types de services publics se mettent en place : dans l'enseignement secondaire, dans l'enseignement supérieur, et pour la formation professionnelle des adultes.

Concernant l'enseignement scolaire, c'est à la fin des années 60 que se concrétisent les choix organisationnels, notamment par la transformation du conseil en orientation en service public national intégré aux appareils de formation ${ }^{3}$ et régulé par des professionnels essentiellement formés à la psychologie. Les CIO (centres d'information et d'orientation) sont créés au sein de l'Éducation nationale, en 1971. Les personnels de l'orientation disposent, dans ces organisations, d'une grande autonomie. L'État y est peu interventionniste. Leurs pratiques professionnelles auront deux constantes : l'approche psychologique et la défense d'une orientation au service de l'émancipation des individus. Par la suite, les pouvoirs publics seront souvent conduits à entériner ces pratiques ou à céder face à la revendication de principes déontolo-

\footnotetext{
${ }^{2}$ La relecture historique des services s'appuie, pour les trois premières phases, sur Danvers (1988, 1999), Caroff (2001), Guichard et Huteau (2007).

${ }^{3}$ Ce choix organisationnel fait suite à une évolution entamée en 1950. À cette date, l'orientation entre à l'école dans le but de gérer les flux d'élèves : elle devient « scolaire ». Avant 1950, l'orientation était « professionnelle » et délivrée dans des organisations très variées par des professionnels formés à l'INOP (Institut national de l'orientation professionnelle), créé en 1928 et ancêtre de l'actuel INETOP (Institut national d'étude du travail et d'orientation professionnelle).
} 
giques $^{4}$. Néanmoins, diverses réformes des services d'orientation scolaire ont été tentées. L'une d'elles, en 1970, visait à réduire le volet «psychologisant" et à rééquilibrer le poids des fonctions de conseil et d'information. Ainsi en 1970, l'ONISEP (Office national d'information sur les enseignements et les professions) se substitue au BUS (Bureau universitaire de statistiques) «pour rassembler et dispenser l'information auprès des publics et établir des prévisions grâce au Céreq (Centre d'études et de recherche sur les qualifications) » (Chiaverini I., Daste P., 1971). Le Céreq est également créé à cette date au sein de l'ONISEP.

Concernant l'enseignement supérieur, chaque université est dotée, en 1975, d'une cellule d'information et d'orientation. Cette initiative s'insère dans le contexte d'universités principalement accessibles aux élites et préparant à des métiers ciblés (médecine, droit, enseignement-recherche...). L'orientation remplit certes une fonction stratégique, mais elle n'agit que sur de faibles volumes. Sa fonction est principalement, alors, une répartition plus harmonieuse des flux entre les spécialités.

Enfin, pour la formation professionnelle des adultes, le fait marquant est la création, en 1966, de l'AFPA (Association nationale pour la formation professionnelle des adultes) ${ }^{5}$ et deux missions qui lui sont confiées : celle de la formation qualifiante des adultes, délivrée dans ses propres centres de formation ; celle de l'orientation, délivrée dans des centres psychotechniques, par des psychologues du travail.

À travers ces différentes réformes, ce qui se joue pour l'orientation, durant ces deux décennies, ce sont avant tout le développement de la scolarisation dans le secondaire et l'adaptation des qualifications aux postes de travail. Dès lors, l'orientation s'inscrit dans la vision politique de planification de l'économie et du marché du travail de l'époque. Cette conception d'une adéquation stricte entre formation et emploi, adaptée

\footnotetext{
${ }^{4}$ Un exemple de recul des pouvoirs publics face à la profession, certes plus tardif, mais qui puise son explication dans cette histoire, est la création par décret du statut de COP (Conseiller d'orientation psychologue), en 1991 (Sénécat, 2004) : décret et arrêté du 20 mars 1991 et loi du 25 juillet 1985 relative au titre de psychologue.

5 Elle remplace l'ANIFRMO (Association nationale interprofessionnelle pour la formation rationnelle de la main-d’œuvre), créée en 1949, pour développer la formation accélérée des adultes après la guerre. Sa gestion est tripartite.
}

à un contexte de faible taux de chômage et de pénurie de main-d'œuvre qualifiée, va être remise en cause par le premier choc pétrolier et la crise économique qui va suivre.

\section{La généralisation (années 1980)}

Les années 80 marquent un tournant dans le positionnement de l'orientation au sein de l'action publique. En effet, les politiques de scolarisation des décennies précédentes ont porté leurs fruits. Les individus entrent mieux formés sur le marché du travail. Les entreprises ont accès à une main-d'œuvre éduquée. Le problème est désormais celui de la réduction d'un chômage de masse qui touche fortement les jeunes à l'entrée dans la vie active et certaines populations à risque, non qualifiées, seniors ou handicapés. Des solutions sont recherchées dans des politiques d'insertion sociale et professionnelle ciblées sur ces catégories ; l'orientation va ainsi devenir un outil au service de ces politiques, conduisant à sa "dilution » au sein d'une multiplication d'intervenants se réclamant de l'orientation, tant des jeunes que des adultes (Guichard et Huteau, 2007).

L'orientation reste à cette époque une des compétences des deux acteurs du noyau dur du service public de l'emploi, l'AFPA et l'ANPE (Agence nationale pour l'emploi). Bien que l'ANPE ait en charge le placement des chômeurs, elle met également en place des dispositifs spécifiques d'orientation vers l'emploi et recourt à l'AFPA pour ce qui est de l'orientation en formation, dans un partage des tâches au demeurant pas toujours très clair. Mais l'orientation se déploie aussi chez tous les intervenants en charge de la mise en œuvre des dispositifs de lutte contre le chômage : les missions locales et $\mathrm{PAIO}^{6}$ (Permanences d'accueil d'information et d'orientation) jeunes qui se développent à partir de 1982 ; le réseau des Cap Emploi, ${ }^{7}$

\footnotetext{
6 Les missions locales et PAIO assurent l'accueil et l'accompagnement des jeunes de 16 à 25 ans, sortis du système scolaire et confrontés à des difficultés d'insertion dans l'emploi. Par leurs fonctions d'accueil, d'information, d'insertion et de suivi - celui-ci pouvant concerner tant la formation que la santé, le logement, les ressources ou les loisirs des jeunes - ces structures proposent un accompagnement global et personnalisé pour l'élaboration d'un parcours individualisé pour chaque jeune.

7 Composé de plus d'une centaine d'organismes de placement, qui regroupent actuellement plus d'un millier de professionnels, Cap Emploi est un réseau réparti sur l'ensemble du territoire français et dont le financement est assuré par l'AGEFIPH (Agence de gestion du fonds d'insertion des personnes handicapées). Conçues comme
} 
Tableau 1

\section{Les services d'orientation en France}

\begin{tabular}{|c|c|}
\hline $\begin{array}{l}\mathrm{ClO} \\
\text { SUIO }\end{array}$ & $\begin{array}{l}3757 \text { COP. } \\
518 \text { directeurs de } \mathrm{ClO} \text {. } \\
\text { Environ } 1000 \text { personnels administratifs des } \mathrm{ClO} \text {, remplissant, entre autres fonctions, } \\
\text { celle de personnel d'accueil. } \\
\text { Pilotage : } 117 \text { inspecteurs de l'Éducation nationale chargés de l'orientation, conseillers } \\
\text { techniques des inspecteurs d'académie en département ; } 30 \text { chefs des services acadé- } \\
\text { miques d'information et d'orientation (CSAIO) et conseillers techniques des recteurs. }\end{array}$ \\
\hline
\end{tabular}

Sous tutelle Travail/Orientation professionnelle

\begin{tabular}{|l|l|}
\hline AFPA & $\begin{array}{l}800 \text { psychologues du travail } 70 \% \text { d'orientation, 10\% d'accompagnement des transi- } \\
\text { tions professionnelles, } 20 \% \text { de suivi psychopédagogique des stagiaires en formation et } \\
\text { conseil en recrutement et gestion des emplois et compétences), appuyés par 200 agents } \\
\text { techniques d'orientation (30\% information et 70\% d'appui administratif et technique). } \\
22 \text { directeurs de Centres régionaux d'orientation professionnelle, assistés de } 45 \text { chargés } \\
\text { de direction responsables de l'orientation. }\end{array}$ \\
\hline ANPE & $\begin{array}{l}1300 \text { agences locales de l'emploi et } 27631 \text { collaborateurs. } \\
\text { Trois métiers : directeur, cadre opérationnel et conseiller polyvalent. }\end{array}$ \\
\hline APEC (Cadres) & Environ 400 consultants en mobilité dans 48 centres. \\
\hline $\begin{array}{l}\text { APECITA } \\
\text { (Cadres agriculture) }\end{array}$ & Plus de 20 délégués régionaux et conseillères emploi-formation. \\
\hline $\begin{array}{l}\text { Missions locales et PAIO } \\
\text { Ueunes) }\end{array}$ & $\begin{array}{l}10 \text { O00 personnes. } \\
70 \% \text { des effectifs exercent une activité d'orientation sur trois métiers : chargé d'accueil, } \\
\text { conseiller, chargé de projet. }\end{array}$ \\
\hline $\begin{array}{l}\text { CAP EMPLOI } \\
\text { (Handicapés) }\end{array}$ & $\begin{array}{l}119 \text { organismes de placement spécialisé. } \\
1100 \text { professionnels spécialisés de l'accompagnement des personnes handicapées vers } \\
\text { l'emploi. }\end{array}$ \\
\hline Établissements interinstitutionnels et paritaires/Orientation professionnelle \\
\hline CIBC & 1300 conseillers bilan de compétences. \\
\hline PRC/PIC-VAE & 800. \\
\hline FONGECIF & 200 à 220 conseillers en mobilité professionnelle. \\
\hline
\end{tabular}

Source : Centre Inffo (2007) complétée par Borras et Campens.

Sigles : CIO : Centres d'information et d'orientation ; SUIO : Services universitaires d'information et d'orientation ; COP : conseillers d'orientation psychologues ; AFPA : Association nationale pour la formation professionnelle des adultes ; ANPE : Agence nationale pour l'emploi ; APEC : Association pour l'emploi des cadres ; APECITA : Association pour l'emploi des cadres, ingénieurs et techniciens dans l'agriculture et l'agro-alimentaire ; ML-PAIO : missions locales-permanences d'accueil d'information et d'orientation ; CIBC : Centres interministériels de bilan de compétences ; PRC/PIC-VAE : Point relais conseil et Points d'information conseil sur la VAE (validation des acquis professionnels) ; FONGECIF : Fonds de gestion des congés individuels de formation. 
qui se structure, après la loi de 1987, sur l'emploi des travailleurs handicapés; les services insertion des conseils généraux ${ }^{8}$, suite à la création du RMI (revenu minimum d'insertion) en 1988.

Que ce soit au sein des missions locales, des PAIO, des Cap Emploi ou des services d'insertion, le conseil en orientation est intégré à une prestation globale d'accueil, d'information, de formation, d'aide au projet, de santé ou de logement, dont la finalité est l'accès à l'emploi. On ne peut décompter les effectifs dédiés à la mise en œuvre de ces missions publiques d'orientation, celle-ci n'étant, pour ces structures, qu'une compétence parmi d'autres. Ceci constitue une première différence par rapport aux services spécialisés de la période précédente. Par ailleurs, nombre de dispositifs développés au cours de la période sont adossés à des bases territoriales. C'est le cas des missions locales et des PAIO, guichets uniques de proximité pour les jeunes, financés en partie par les collectivités locales. Cette implantation territoriale de l'orientation est une seconde différence notable comparativement aux choix organisationnels antérieurs de services publics nationaux.

Le paysage de la politique de l'emploi ayant évolué vers une plus grande complexité institutionnelle et le conseil en orientation aux demandeurs d'emplois ayant été intégré à tous les dispositifs de la politique de l'emploi, il est, de ce fait, également dispersé, peu visible et non coordonné.

\section{L'amorce d'une privatisation (années 1990)}

Les années 90 sont le théâtre de nouvelles inflexions de l'action publique en matière d'orientation. Son rôle change au sein du monde éducatif où une nouvelle conception, basée sur « l'éducation à l'orientation », re-légitime les approches centrées sur les individus.

un service de proximité, les institutions de ce réseau ont pour vocation d'aider les entreprises et les personnes handicapées dans leurs démarches, soit de recrutement soit de construction d'un parcours vers l'emploi.

8 À la suite de la loi du 16/12/2003 décentralisant le RMI et créant le RMA (Revenu minimum d'activité), les départements ont été conduits à redéfinir leurs politiques d'insertion envers les bénéficiaires du RMI. Sur ce registre, les conseils généraux ont désormais pour mission d'organiser l'orientation des bénéficiaires vers des « référents » chargés de les accompagner dans leur parcours d'insertion.

9 Selon les termes de la circulaire $n^{\circ} 96-230$ du $1^{\text {er }}$ octobre 1996, l'éducation à l'orientation se définit comme une démarche
Cependant, les transformations les plus notables, au cours de cette période, concernent le monde du travail. L'orientation devient un vecteur d'une normalisation de l'accompagnement des mobilités professionnelles. Des dispositifs (le bilan de compétence et la validation des acquis de l'expérience) sont définis par les partenaires sociaux dans le cadre du financement de la formation professionnelle continue. Ils sont en partie mis en œuvre par des prestataires publics, mais de plus en plus par des organismes privés agréés par l'administration.

Un constat est à l'origine de ces inflexions. Les politiques d'insertion n'ont pas suffi à réduire le chômage de masse. Les réponses politiques sont de plus en plus recherchées du côté des entreprises, soumises à de fortes contraintes d'adaptabilité afin de faire face à une concurrence exacerbée au plan mondial. Dans ce cadre, l'orientation est mobilisée pour accroître l'employabilité des salariés et elle va prendre la forme principale de deux dispositifs complémentaires : les bilans de compétence (BDC) pour l'aide au projet professionnel et la validation des acquis de l'expérience (VAE) ${ }^{10}$ pour la reconnaissance des acquis ${ }^{11}$ (Céreq, 2006).

Le développement de ces deux dispositifs va générer une réorganisation de l'offre de services et de conseil en orientation. Le marché s'ouvre et la création d'organismes privés répondant aux besoins individuels s'intensifie. À côté de la composante institutionnelle publique, comme les centres interinstitutionnels de bilans de compétences (CIBC) ${ }^{12}$, coexiste désormais une composante privée. De cette nouvelle donne de l’offre découle une hétérogénéité des pratiques, marquée par une forte mobilité des professionnels exerçant dans ces structures et une précarisation de leur statut pour au moins un tiers d'entre eux (Dares, 2005).

engageant la responsabilité individuelle, favorisée par l'institution éducative : "La décision d'orientation doit rester de la responsabilité personnelle du lycéen et de l'étudiant. Ce choix doit être préparé par une éducation et une information approfondies ».

${ }^{10}$ Le BDC est instauré par la loi du 31/12/1991 et la VAE par la loi de modernisation sociale du 18/01/2002.

11 On note également le développement d'un conseil en orientation des salariés au sein des FONGECIF (Fonds de gestion du congé individuel de formation), mais les effectifs concernés sont très faibles. Voir tableau 1.

${ }^{12}$ Institués par la circulaire du 14 mars 1986 du ministère du Travail. 
À l'issue de ces quarante années, trois segments de l'orientation - jeunes, demandeurs d'emplois et salariés - sont constitués. Ces trois segments fonctionnent de manière cloisonnée, à la fois en raison de publics et de financements distincts mais aussi du fait de statuts et de qualifications de leurs personnels très hétérogènes (Centrinffo, 2007).

\section{L'européanisation du cadre national (années 2000)}

En France, les années 2000 ouvrent à nouveau la voie à des transformations significatives de l'action publique de l'orientation, sous l'influence de diverses initiatives communautaires et de l'OCDE visant à instaurer une formation puis une orientation tout au long de la vie.

En mars 2000, l’Union européenne engage une série de réformes de ses politiques de formation et d'emploi dans le but de réaliser l'objectif, à dix ans, fixé lors du Conseil européen de Lisbonne, qui est de « devenir l'économie de la connaissance la plus compétitive et la plus dynamique du monde ». Les politiques d'orientation professionnelle prennent peu à peu une place centrale dans la conduite de ces réformes et un certain nombre de préconisations dans ce sens sont avancées, en 2004, dans un rapport conjoint avec l'OCDE (2004-a) « Career Guidance. Handbook for Policy Makers » («L'orientation professionnelle: guide pratique pour les décideurs »). Le Cédefop est le relais des politiques européennes (Cedefop, 2004 et 2005).

L'OCDE publie pour sa part, en 2004, un rapport (2004-b) devenu depuis une référence « Orientation professionnelle et politique publique, comment combler l'écart? » et qui fait suite à de premiers travaux $(2001,2002)$ ayant posé les termes d'un débat sur la qualité et le pilotage de l'orientation. Le rapport de 2004, basé sur les résultats d'un examen des politiques d'orientation professionnelle dans quatorze pays, cherche à répondre à une question : comment l'organisation et le fonctionnement des services d'orientation contribuent-ils à la réalisation d'objectifs des politiques publiques? Deux conclusions s'en dégagent. En premier lieu, l'orientation est désormais qualifiée de « tout au long de la vie ». Si elle concerne encore les jeunes en scolarité, une inflexion de l'action est nécessaire pour prendre en charge les adultes, de plus en plus confrontés à des mobilités en cours de vie active. En second lieu, les débats sur la modernisation des services publics en Europe atteignent aussi les services d'orientation, mettant en cause les choix organisationnels passés. Au plan communautaire, d'autres rapports suivront, commandés par l'Union européenne (Sultana et Watts, 2005) ou ayant bénéficié de subventions communautaires (Frade et alii, 2005 ; Personnaz et alii, 2007 ; Darmon et alii, 2007 ; Cuntigh et alii, 2006). Ces derniers sont focalisés sur l'orientation des adultes et l'orientation au sein du service public de l'emploi.

Bien qu'absente de l'examen des pays de l'OCDE, la France entreprend, dans le même temps, une réflexion sur son action en matière d'orientation. Des rapports gouvernementaux sur l'orientation se multiplient (Henoque, Legrand, 2004 ; Tharin, 2005 ; MENESR, 2007 ; Reiss, 2007) mais ne considèrent essentiellement que l'orientation scolaire, son fonctionnement, son évaluation et son articulation avec les politiques éducatives ; l'orientation des adultes demeure marginale dans les débats. Ceci s'explique en partie par un double contexte. Tout d'abord, le questionnement sur l’orientation est en France ravivé par les difficultés massives d'insertion des jeunes et l'échec scolaire, qui trouveront une expression sociale et médiatique forte lors des émeutes urbaines de novembre 2006. À la même période, l'actualité place sur le devant de la scène la question du transfert des COP aux Régions.

Les diverses initiatives et recommandations formulées par les instances communautaires et internationales ont des incidences indéniables sur la conception et le pilotage des politiques d'orientation dans les différents pays. L'une des conséquences est la prise de conscience de la nécessité de réformer les dispositifs nationaux d'orientation et d'envisager leur décloisonnement afin de rendre opératoire la notion préconisée «d'orientation tout au long de la vie » (OTLV). Si la France est un cas emblématique de système d'orientation segmenté, dans la plupart des pays de l'OCDE et de l'Union européenne, une telle segmentation prévaut aussi. La levée de cet obstacle institutionnel et politique devient un élément-pivot des réformes nationales qui s'engagent. 


\section{LA QUALITÉ ET LA SEGMENTATION DES SERVICES D'ORIENTATION DÉBATTUES EN FRANCE...}

Le mouvement de réforme, en France, se traduit par des évolutions à deux niveaux. Des réformes transversales aux trois segments de l'orientation sont conduites au plan régional, en lien avec les différentes lois de décentralisation ${ }^{13}$ (Borras et alii, 2008). Les premières initiatives datent de 1993. Dans le même temps, des réformes internes à chaque segment continuent à être menées parallèlement au niveau central.

\section{Décloisonnement et qualité : des chantiers ouverts au niveau régional}

En dépit d'un domaine de compétence très restreint, les pouvoirs publics régionaux vont être les initiateurs d'une série d'innovations en matière de définition des politiques d'orientation et de coordination des acteurs au plan territorial. Cette prise en main du sujet s'explique probablement par le fait que les pouvoirs publics régionaux sont confrontés directement aux attentes des publics et des territoires et contraints à élaborer des réponses opérationnelles.

Parmi les expérimentations repérables, peut être évoqué le cas de quelques régions qui, dès 1993, se préoccupent d'orientation dans le cadre de la préparation de leur plan régional de développement des formations (PRDF). Une réflexion conjointe entre partenaires sociaux, État et Régions dressera un bilan de ces politiques régionales dans le champ de l'orientation : «Le système français d'AIO (Accueil, information, orientation) apparaît comme un ensemble de dispositifs ne parvenant pas à se constituer en secteur doté de normes, de valeurs et de pratiques communes »; il y a urgence à «poursuivre l'effort de clarification et de simplification annoncé par la loi quinquennale de 1993 afin que chaque citoyen puisse disposer à proximité sur les territoires d'un accompagnement de qualité de ses démarches individuelles,

13 1982, 1993, 2002... La loi du 13/08/2004 réaffirme l'entière responsabilité des Régions sur l'apprentissage et la formation professionnelle des jeunes et des adultes à la recherche d'un emploi (dès lors que ces formations ne relèvent pas de l'assurance chômage et de l'entreprise). Leur champ d'intervention est étendu à l'organisation du réseau des centres et points d'information et de conseil sur la VAE. autrement dit d'une orientation active allant bien au-delà de l'information dispensée par un guichet institutionnel » (CCPR - Comité de coordination des programmes régionaux -, 2004). Ces constats se rapprochent de la conception communautaire de l'orientation et débouchent également sur les enjeux de coordination des services et de leur qualité. Ce sont les actions de ces régions pionnières qui s'étendront progressivement aux autres régions françaises.

Ainsi, en matière de coordination régionale, la région Rhône-Alpes devient précurseur avec la mise en place, en 2004, du Pôle Rhône-Alpes de l'orientation (PRAO), chargé de l'amélioration et du développement des services d'AIO. Le PRAO propose aujourd'hui à ses vingt et un réseaux adhérents des outils " communs et fédérateurs », définis à partir d'une analyse des besoins des publics. Autre exemple, l'Ile-de-France est engagée dans une démarche similaire de coordination par une instance régionale, le CARIF (Centre d'animation, de ressources et d'information sur la formation), en charge, depuis 2002, de l'information sur les métiers, la professionnalisation des acteurs et leur mise en réseau. Une dernière illustration est fournie par les centres de ressources et d'information des professionnels et des publics du Réseau Nouvelle Chance en Aquitaine. ${ }^{14}$

Les Régions s'attellent aussi au chantier de la qualité des services (Cheynet, Normand, 2005). Ce terme apparaît dans les diagnostics et programmes d'action établis à la demande de plusieurs conseils régionaux par le cabinet Amnyos (2006 a- et b-). On peut citer, dans ce domaine, le cas de la Bourgogne qui envisageait, en 2006, la mise en place d'une charte fédérative en vue d'améliorer la qualité et l'efficacité de l'AIO ; le cas de Rhône-Alpes avec le projet " $\mathrm{DROA}^{15}$ ", qui débouche, en 2006, sur un « référentiel qualité de l'orientation » propre à favoriser un meilleur accompagnement professionnel tout au long de la vie; le

\footnotetext{
14 S'inscrivant dans le cadre de la mise en place d'expérimentations de la formation professionnelle (circulaire DGEFP n ${ }^{\circ}$ 2002/8 du 21/03/2002), cette structure aquitaine vise la mise en réseau des services d'accueil, d'information et d'orientation en vue de constituer des centres de ressources pour les acteurs régionaux de l'information.

15 Projet conduit entre 2003 et 2006, dans le cadre d'un programme européen Léonardo da Vinci, en partenariat avec quatre régions d'Europe. Il consistait à comparer les organisations et pratiques locales d'orientation.
} 
cas de Languedoc-Roussillon visant à développer une approche régionale de la qualité des services.

Au-delà de cette réalité foisonnante d'actions dans les régions, force est de constater, malgré tout, que la prise de responsabilité sur le champ de l'orientation a été lente et différenciée selon les régions (CCPR, 2002). Sans doute que les moyens financiers et légaux ont été longs à se mettre en place. En outre, malgré l'obligation faite aux conseils régionaux de produire un «plan d'action pour la mise en œuvre d'une politique d'information et d'orientation au sein du PRDFPJ (Plan régional de développement de la formation professionnelle des jeunes) » (art. 53), la loi quinquennale de 1993 a laissé planer une grande ambiguïté au terme de son article 49 : «La Région aura compétence pour l'ensemble de la formation professionnelle continue en faveur des jeunes de moins de vingt-six ans et disposera à ce titre des compétences exercées par l'État en matière de formation professionnelle sur le réseau d'accueil, d'information, d'orientation et de suivi des jeunes. » Autrement dit, pour les publics jeunes, les Régions auraient compétence pour l'orientation en formation, tandis que l'État garderait celle des activités des missions locales et PAIO ayant trait à l'insertion. Dans le partage de la « relation formationemploi »- qui délègue aux conseils régionaux une attribution générale en matière de formation professionnelle et conserve à l'État la maîtrise de la politique de l'emploi - la question des fonctions d'orientation n’a pas été vraiment tranchée et demeure aujourd'hui en suspens (Berthet, Gayraud, 2003).

\section{Des initiatives régionales peu relayées au niveau central}

Au-delà de ces expériences régionales, des initiatives sont prises au plan national. Après l'échec du transfert aux conseils régionaux de l'animation du réseau des COP et la crise des banlieues de l'automne 2006, le Premier ministre nomme un Délégué Interministériel à l'Orientation (DIO). Suite à une mission de réflexion sur la réforme du dispositif national d'orientation, un schéma national de l'orientation est élaboré, en mars 2007. Les principales lignes d'action sont la réduction des sorties sans qualification et le développement d'une meilleure connaissance des métiers et du monde du travail dans l'enseignement secondaire ; la réduction du décrochage en première année à l'université et la facilitation des transitions vers l'emploi pour le supérieur. La « Délégation », bien qu'à vocation interministérielle, met donc en œuvre de premières réformes visant le segment de l'orientation scolaire.

En parallèle, les débats sur l'orientation des adultes sont réactivés, dès 2003, dans le cadre des négociations successives sur la réforme de la formation professionnelle continue et sur le régime d'assurance chômage. Le dernier accord sur la formation, en date du 7 janvier 2009, est suivi d'un projet de loi relatif à l'orientation et la formation professionnelle tout au long de la vie. Son article 3 prévoit d'améliorer la qualité de l'information et de la formation professionnelle des jeunes sortis du système scolaire et des adultes. Un décloisonnement entre les services aux salariés et aux demandeurs d'emploi est donc à attendre, qui aura des conséquences sur les structures et leurs financements, notamment par l'inclusion des services d'orientation de l'AFPA au sein de PôleEmploi. Cette option, qui conduit à intégrer, d'ici 2011, les psychologues de l'AFPA à l'organisme d'indemnisation et de placement, constitue un signal fort concernant la conception de l'orientation dans son articulation aux politiques d'emploi. L'orientation est clairement affirmée comme un instrument de l'activation des demandeurs d'emplois, participant au paradigme d'adaptation des individus au marché du travail, qui découle de la déclinaison de la Stratégie européenne pour l'emploi.

Pour que ces réformes engagées au niveau central, ces dernières années, prennent désormais le relais des initiatives régionales engagées depuis bientôt quinze ans, il faudrait qu'elles se fondent sur une plus grande coopération État-Région. Or, cela est encore rarement le cas. En la matière, les protocoles d'expérimentation de la formation professionnelle, conclus en 1999 par l'État avec sept régions ${ }^{16}$, et qui visaient la mise en réseau des services d'AIO, constituent une exception notable. En outre, l'initiative toute récente de la DIO et du Haut Commissaire à la Jeunesse, qui travaillent ensemble à produire une circulaire interministérielle concernant le repérage et le traitement des jeunes sans qualification, va dans le même sens. Cette circulaire prévoit de s’appuyer sur les « bonnes pratiques régio-

\footnotetext{
${ }^{16}$ Aquitaine, Centre, Pays de la Loire, Midi-Pyrénées, Réunion, Basse-Normandie, Rhône-Alpes. Circulaire DGEFP n 2002-8 du 21/3/2002.
} 
nales » de coordination des acteurs de l'orientation scolaire et postscolaire. Ce pas en avant vers plus de transversalité constitue une réponse à l'urgence sociale d'offrir aux jeunes des services lisibles, accessibles et efficaces.

\section{... ET EN DÉBATS À l'ÉTRANGER}

Rémanents au sein de l'ensemble des pays de l'OCDE, les deux grands enjeux de politique publique amélioration de la qualité des services d'une part et décloisonnement des segments de l'orientation d'autre part - suscitent dans chaque pays des voies de réformes spécifiques. La mise en perspective des diverses expériences nationales révèle les termes de ces débats actuels. Elle montre également la diversité des solutions recherchées pour répondre à ces deux grands enjeux abordés successivement ci-après.

\section{Des démarches qualité qui percutent les régulations professionnelles}

Les diagnostics sur la nécessité d’améliorer la qualité des services convergent dans la plupart des pays : les enjeux portent sur un meilleur usage des TIC (Technologies de l'information et de la communication), une réduction de l'approche psychologique et un développement de l'information, des services aux adultes et un meilleur ciblage des services... (OCDE, 2004-b). En revanche, des divergences apparaissent dans les solutions envisagées. Dans son rapport de 2001, l’OCDE explore deux pistes. La première privilégie la définition, par les professionnels, de référentiels de compétences et de codes de déontologie, confortant par là même les régulations professionnelles des services. La seconde prend appui sur la création de standards nationaux de qualité, de procédures d'accréditation et de mise en concurrence des prestataires publics et privés. L’exemple le plus avancé dans la définition de référentiels de compétences est fourni par le Canada qui promeut, au début des années 2000, une amélioration de la qualité par la mise au point de référentiels de compétences professionnelles. Ainsi un « Guide canadien pour les professionnels en développement de carrière » a été élaboré grâce à une large implication de l'ensemble des professionnels dans une démarche concertée. Ce Guide a connu une forme de généralisation car il a inspiré les normes internationales mises au point par l'Association internationale d'orientation scolaire et professionnelle (AIOSP). Cette association de professionnels a pour mission de promouvoir, au plan international, des services d'orientation de qualité17. Le référentiel de l'AIOSP a été ratifié par un groupe de 700 praticiens de trentehuit pays, en 2003.

Une voie alternative au choix précédent est celui des normes de qualité et d'accréditation. L'exemple le plus avancé est développé, à la fin des années 90, au Royaume-Uni (OCDE, 2004-b). Dans ce pays, le National Information Advice and Guidance Board ${ }^{18}$ coordonne l'orientation professionnelle par l'intermédiaire du ministère de l'Éducation et des Qualifications et du ministère du Travail et des Retraites. Un autre organisme, le Guidance Council, définit des normes de qualité des multiples acteurs de l'orientation. Afin de s'assurer de la réalisation de ces recommandations, les organismes chargés d'effectuer l'orientation professionnelle ne sont subventionnés qu'à la condition qu'ils respectent les standards. En parallèle, un programme de formation a été créé à l'University of East London qui propose, depuis le début des années 2000, un diplôme de troisième cycle en orientation professionnelle tous publics. Cette évolution s'insère dans des pratiques de réforme en Europe, plus caractéristiques des pays anglo-saxons, et consistant à importer dans les services publics des instruments issus du privé. Repérées sous le terme de «nouvelle gestion publique ", ces réformes poursuivent deux objectifs : mieux définir le service à partir des besoins des usagers et non en fonction des contraintes des prescripteurs ; faire en sorte que les services publics bénéficient prioritairement aux publics en ayant le plus besoin. Sur ce second objectif, il semble qu'en effet les services publics, en raison de leur gratuité et de leur accessibilité à tous, profitent souvent aux publics qui en auraient le moins besoin.

Les régulations professionnelles de l'orientation sont donc interrogées, au plan international, par le développement des mécanismes marchands. Mais l'avenir

\footnotetext{
17 http://www.iaevg.org

18 « Service national d'information, de conseil et d'orientation » (traduction des auteurs).
} 
semble ouvert, la pertinence des outils de la nouvelle gestion publique pour l'orientation suscitant de vifs débats, y compris au sein de l'OCDE. Les arguments avancés (2001, 2002, 2004-b) sont nuancés : certains rapports insistent sur la rareté des évaluations régulières et systématiques de la qualité des services ; d'autres pointent la difficulté de développer une mesure fiable de l'impact des services d'orientation.

Plus globalement, les réflexions sur la qualité des services ont lieu avec en filigrane la contrainte budgétaire et le contrôle des dépenses publiques. La dualisation de l'offre de services de l'Agence Connections, au Royaume-Uni, est un exemple parmi d'autres de réponse à cette contrainte. Cette agence a mis en place des services en ligne téléphoniques, 24h/24h et tous publics, élargissant ainsi l'accès au service à distance tout en réservant le face-à-face, plus coûteux, à des publics particuliers. De même, le décloisonnement institutionnel est source d'économies de divers points de vue. La création de services tous âges permet théoriquement de rééquilibrer le poids des services aux jeunes et aux adultes par une gestion des emplois plus souple. Sans cette contrainte, les services spécialisés, régulés par les professionnels, ne pourraient-ils s'étoffer et perdurer ? Par ailleurs, initier une réflexion sur des services tous âges conduit à remettre en cause la diversité des choix organisationnels, la coexistence de services publics et privés, plus ou moins spécialisés ou intégrés à des services d'accompagnement. Ceci ne manquerait pas de soulever de fortes résistances statutaires, mais aussi des interrogations sur les liens entre qualité du service et compétences professionnelles. La spécificité des problèmes d'emploi des jeunes et des adultes ne suppose-t-elle pas un certain degré de spécialisation, garant d'une qualité de service ?

\section{Le décloisonnement des segments de l'orientation : vers une nouvelle politique?}

Concernant le décloisonnement institutionnel, diverses solutions sont expérimentées à l'étranger (OCDE, 2004-b). Plus ou moins centralisées, elles s’appuient sur des instances infranationales et conduisent, selon les cas, à la mise en place de guichets uniques locaux de services tous publics. Quelques exemples illustrent ces tentatives :
L'Angleterre, comme souligné précédemment, a privilégié une mise en cohérence des politiques d'orientation par un seul ministère. D’autres pays, souvent à tradition décentralisée, privilégient des instances régionales. En Espagne, un conseil général pour l'apprentissage - regroupant le ministère de l'Emploi et des Affaires sociales, les gouvernements régionaux, les communautés autonomes et les partenaires sociaux est chargé d'assurer la coordination des politiques et de poser les bases d'un système intégré d'orientation. Ces expérimentations ne sont pas toujours probantes, car la coordination ne naît pas de simples recommandations, et malgré le principe d'égalité de traitement, des disparités territoriales apparaissent. Ce problème peut être contourné par la mise en place conjointe de commissions nationales. Career Wales, service interâges d'orientation professionnelle, créé en 2001 au Pays de Galles, témoigne d'une autre voie possible. Cette confédération, composée de sept entreprises régionales rassemblées sous une appellation commune, est responsable des services destinés aux jeunes, de l'orientation professionnelle des adultes, du réseau learndirect de centres d'appel, du Youth Gateway (un cours bref et intensif d'acquisition des compétences nécessaires au passage à la vie active conçu à l'intention des jeunes à risque âgés de 16/17 ans) et des rapports entre l'éducation et l'entreprise. Il soutient les programmes d'orientation professionnelle organisés dans les écoles et les collèges. Ses services sont ouverts sans rendez-vous aux personnes de tous âges. Des services aux adultes sont organisés par prestations itinérantes, par téléphone ou en ligne. Si le personnel de Career Wales travaille avec des jeunes et des adultes, il est en général spécialisé.

Ces évolutions institutionnelles nationales sont de nature à transformer profondément les liens entre orientation et politiques publiques. Développer des services unifiés d'orientation signifie aussi éloigner, voire sortir l'orientation des appareils de formation, des dispositifs de la politique de l'emploi et de la formation continue. Ceci conduit à réduire son rôle d'instrument de régulation des flux dans ces systèmes. L'orientation est traditionnellement située au confluent des logiques individuelles et des régulations institutionnelles (Berthet T., Grelet, Y., Romani C., 2008). L’orientation tout au long de la vie et le décloisonnement invitent à un rééquilibrage au profit des logiques individuelles, 
principe somme toute en concordance avec l'approche promue par l'Union européenne de services « accessibles à tous en permanence, qui gomment la distinction entre orientation scolaire, professionnelle et personnelle [...] et qui cessent d'être axés sur l'offre pour s'orienter sur la demande et placer les besoins et les exigences des utilisateurs au cœur de leurs préoccupations » (Union européenne, 2000).

Après quinze années d'initiatives régionales et de fortes incitations de l'Union européenne, les réformes institutionnelles de l'orientation, en France, émergent en 2009. Les problèmes de la transversalité des services et de la qualité commencent désormais à être abordés ; l'idée d'un grand service public de l'orientation, qui engloberait l'initial et le « tout au long de la vie ", progresse, notamment dans le cadre de la nouvelle loi sur l'orientation et la formation professionnelle. La qualité des services passe d'un stade de réflexion à un stade plus opérationnel. Un chantier s'ouvre actuellement sur la définition de normes de qualité qui devrait conduire, d'une part, à préciser les missions du service d'information et d'orientation et d'autre part, à mettre en place des procédures d'évaluation de son efficacité.

Ces dernières avancées soulèvent trois questions. La première concerne la mise en place d'une nouvelle architecture de l'action publique en matière d'orientation. En effet, l'État s'empare désormais du sujet de manière volontariste, reprenant un certain leadership stratégique dans le domaine. Ce repositionnement implique une nouvelle répartition des responsabilités entre l’État et les conseils régionaux, devenus incontournables pour le pilotage de l'orientation dans les territoires. La deuxième question a trait à la place qui devrait être attribuée aux usagers, tant du côté des employeurs, largement absents jusqu'alors des régulations de l'orientation, que des représentants des jeunes, des familles ou encore des salariés et des demandeurs d'emploi. Quelle voix peuventils et doivent-ils apporter à la définition des futures normes de qualité ? Sur ce point, les associations internationales de professionnels sont sensibles au développement d'une implication "citoyenne». Celle-ci est de nature à renforcer une autre conception du conseil en orientation : les conseillers n'orientent pas en faisant les choix «à la place de ...»; ce sont les citoyens qui s'orientent, éclairés par un conseil de qualité. La troisième question renvoie au rôle des professionnels dans cette nouvelle architecture institutionnelle et en tant que premiers garants de la qualité des services. Compte tenu de leur rôle historique dans les régulations de l'orientation en France, cette dernière question est lourde d'enjeux pour la mise en œuvre des réformes à venir.

Pour objectiver la réflexion autour de ces questions cruciales, souvent sujettes à antagonismes idéologiques, les apports de la recherche semblent ici plus que jamais nécessaires. Au-delà de l'approche psychologique, devenue prédominante dans les études sur l'orientation, il s'avère nécessaire de multiplier les approches, de croiser les disciplines et d'investir plus fortement le champ de l'orientation des adultes. Il y a également urgence à développer à nouveau les approches institutionnelles et à enrichir les comparaisons internationales déjà existantes. Il conviendrait de les poursuivre sous l'angle de l'analyse historique des institutions, indispensable à une meilleure compréhension du sens de l'action publique 
Amnyos consultants (2005), Rhône-Alpes. Évaluation des modalités de contractualisation entre l'État, la Région et les branches professionnelles au titre du programme emploi, formation et économie des contrats de plan État-Région, (CPER).

Amnyos consultants (2006 a), L'offre de services d'AIO dans le cadre de l'élaboration du PRDF de la région Languedoc-Roussillon.

Amnyos consultants (2006 b), Assistance à la mise en place d'un système coordonné d'AIO en Bourgogne.

Berthet T., Gayraud L. (2003), « Gouverner l'action publique aux marges : le cas des fonctions d'accueil, d'information et d'orientation », in M. Bel, P. Méhaut, O. Mériaux, La décentralisation de la formation professionnelle, quels changements dans la conduite de l'action publique ?, Paris, L'Harmattan.

Berthet T., Grelet Y., Romani C. (2008), « Le système d'orientation », Nef Céreq nº 6, octobre 2008.

Borras I. (2004), « L'orientation des jeunes peu qualifiés, une liberté contrainte », Formation Emploi, $n^{\circ} 88$.

Borras I. (collab.) Berthet T., Campens E., Romani C. (2008), « Le pilotage de l'orientation tout au long de la vie. Le sens des réformes ", Nef Céreq n ${ }^{29}$, juillet.

Caroff (2001), L'organisation de l'orientation des jeunes en France. Évolution des origines à nos jours. EAP.

CCPR (2004), Pour une politique régionale coordonnée d'accueil, d'information et d'orientation, Janvier.

CCPR (2002), Évaluation des politiques régionales de formation professionnelle initiale et continue, Rapport d'évaluation exercice 2000-2002.

Cedefop (2004), Les politiques de l'orientation dans la société de la connaissance. Tendances, défis et réponses en Europe, Luxembourg, Office des Publications Officielles des Communautés européennes, panorama séries n 85 .

Cedefop (200), Améliorer les politiques et les systèmes d'orientation tout au long de la vie, en utilisant les outils de référence européens, Office des Publications Officielles des Communautés européennes.

Centre inffo (2007), «La professionnalisation des acteurs de l'orientation », Actualité de la formation permanente, $\mathrm{n}^{\circ} 208$.

Céreq, (1999), L'orientation professionnelle des adultes, collection « Études », $\mathrm{n}^{\circ} 73$.

Céreq (2006), « La VAE, quels candidats pour quels diplômes ? », Bref-Céreq, n 230, mai.

Chiaverini I., Daste P. (1971), Orientation scolaire et professionnelle, Berger-Levrault, Paris.

Cuntigh P., Perez C., Personnaz E. (2006), «L'orientation professionnelle des chômeurs en Europe », Bref-Cereq, n 232.

Danvers F. (1988), Le conseil en orientation en France, de 1914 à nos jours, Paris, EAP.

Danvers F. (1999), "L'orientation: perspective historique ", in Cereq, L'orientation professionnelle des adultes, contributions de la recherche, état des pratiques, AFPA, n 73, Céreq, Études, 07/1999.

Dares (2005), « Les bilans de compétence : en hausse pour les demandeurs d'emplois, stabilité pour les salariés, " Premières informations, premières synthèses, $\mathrm{n}^{\circ} 07.02$, février.

Darmon I., Perez C. (2007), Evaluation Guide for Information, Advice and Guidance Services for Adults, WP4, Leonardo Programme, Guidance in Europe Project, ES/04/C/F/RF-80900, 45 p.

Frade C., Darmon I., Alvarez I. (2005), Adult Guidance Systems in Five European Countries: A 
Comparative Analysis, WP1 Comparative report, Leonardo Programme, Guidance in Europe Project, ES/04/C/F/RF-80900, 143 p.

Guichard J. et Huteau M. (2007), L'orientation scolaire et professionnelle, Dunod.

Guichard J. et Huteau M. (dir.) (2007), Orientation et insertion professionnelle : 75 Concepts clés, Paris, Dunod.

Hénoque M., Legrand A. (2004), L'évaluation de l'orientation à la fin du collège et au lycée, Rapport pour le Haut Conseil de l'Évaluation de l'École, mars.

MENESR (2005), Le fonctionnement des services d'information et d'orientation, rapport nº 2005101 à Monsieur le Ministre.

Normand R. \& Cheynet P. (2007), « Les confluences de l'orientation tout au long de la vie: La qualité comme instrument de coordination de l'action publique régionale ", in Hélène Buisson-Fenet \& Gwenola Le Naour (dirs.), Les professionnels de l'action publique face à leurs instruments, Paris, Éditions Octarès.

OCDE (2001), L'orientation professionnelle et la qualité, Plant $\mathrm{P}$.

OCDE (2002), An occupation in harmony. The roles of markets and governments in career information and career guidance, Grubb Norton W.

OCDE (2004-a), Career Guidance. Handbook for Policy Makers.

OCDE (2004-b), Orientation professionnelle et politique publique, comment combler l'écart ?
Perez C., Personnaz E. (2008), « Les services d'information, de conseil et d'orientation professionnelle des adultes », Cereq, Relief, ${ }^{\circ} 27$.

Personnaz E., Segas S., Darmon I. (2007), Advice and Guidance services for out of work individuals and workers 'at risk' in five European countries: an exploratory typology (vol. 1), WP3, Leonardo Programme, Guidance in Europe Project, ES/04/C/F/ RF-80900, 102 p.

Reiss F. (2007), Avis présenté à l'Assemblée nationale au nom de la commission des affaires culturelles, familiales et sociales sur le projet de loi de finances pour 2008 ( $\mathrm{n}^{\circ}$ 189). Tome IV. Enseignement scolaire (avis portant sur les six programmes de la « mission » enseignement scolaire).

Seillier B. (2007), Formation professionnelle: le droit de savoir, Rapport du Sénat.

Senécat J. (2004), Historique de l'orientation scolaire et professionnelle en France, extrait de L'orientation en Europe, des approches différentes pour une question commune, Les rencontres de la DESCO, www. eduscol.education.fr.

Sultana R. et Watts A.G. (2005), Career Guidance in Europe's Public Employment Services: Trends and Challenges, Report commissioned by DG Employment, Social Affairs and Equal Opportunities (European Commission).

Tharin I. (2005), Orientation, réussite scolaire: ensemble, relevons le défi!, Rapport au Premier ministre, Paris, La Documentation française.

Union européenne (2000), Mémorandum sur l'éducation et la formation tout au long de la vie, Document de travail des services de la commission, 30.10.2000, SEC(2000) 1832. 
Résumé

\section{Orientation et politiques publiques \\ Évolutions nationales, enjeux internationaux}

Isabelle Borras et Claudine Romani

Cet article traite des évolutions institutionnelles de l'orientation, en France, depuis le début des années 60. Le regard historique porté sur le développement des services d'orientation met l'accent sur leur cohérence avec les politiques éducatives et les transformations du marché du travail. Ce panorama rétrospectif distingue quatre phases qui renvoient à différentes conceptions du rôle de l'orientation. II replace par ailleurs les évolutions récentes en France sur la toile de fond des stratégies de l'Union européenne et de I'OCDE (Organisation de coopération et de développement économiques) pour le déploiement d'une orientation tout au long de la vie requérant une mise en œuvre coordonnée et de qualité. L'article fait référence à des initiatives régionales allant dans ce sens et explique certains obstacles auxquels est confrontée une généralisation des approches expérimentées en France. Enfin, il évoque les différentes options d'action publique prises à l'étranger pour réformer les services d'orientation.

\section{Mots clés}

Orientation scolaire-professionnelle, politique publique, union européenne, étude historique, orientation tout au long de la vie

Journal of Economic Literature: I 21, I 28, O 52 